\title{
Orbitofrontal Cortex Encodes Memories within Value-Based Schemas and Represents Contexts That Guide Memory Retrieval
}

\author{
@Anja Farovik, Ryan J. Place, Sam McKenzie, Blake Porter, Catherine E. Munro, and Howard Eichenbaum \\ Center for Memory and Brain, Boston University, Boston, Massachusetts 02215
}

\begin{abstract}
There are a substantial number of studies showing that the orbitofrontal cortex links events to reward values, whereas the hippocampus links events to the context in which they occur. Here we asked how the orbitofrontal cortex contributes to memory where context determines the reward values associated with events. After rats learned object-reward associations that differed depending on the spatial context in which the objects were presented, neuronal ensembles in orbitofrontal cortex represented distinct value-based schemas, each composed of a systematic organization of the representations of objects in the contexts and positions where they were associated with reward or nonreward. Orbitofrontal ensembles also represent the different spatial contexts that define the mappings of stimuli to actions that lead to reward or nonreward. These findings, combined with observations on complementary memory representation within the hippocampus, suggest mechanisms through which prefrontal cortex and the hippocampus interact in support of context-guided memory.
\end{abstract}

Key words: context; memory; orbitofrontal cortex; rat; retrieval; value

\section{Introduction}

Learning often incorporates the background cues, or context, in which important events occur. Subsequently, the context can be used as a cue to retrieve the memories relevant for goal-directed behavior appropriate to that context (Greenspoon and Ranyard, 1957; Balaz et al., 1981). Here we consider the brain mechanisms that support behavior where context guides the encoding and retrieval of relevant memories.

Previous studies have strongly implicated the hippocampus in the formation and retrieval of memories composed of events and their associated contextual details (Smith and Mizumori, 2006; Komorowski et al., 2009, 2013; Langston and Wood, 2010). Prefrontal cortex has been strongly implicated in the development of abstract contextual organizations and the control of memory retrieval (Miller and Cohen, 2001; Peters et al., 2013). Prefrontal areas and the hippocampal formation are strongly interconnected (Delatour and Witter, 2002; Agster and Burwell, 2009; Kondo and Witter, 2014), suggesting that an exchange of information between these areas might support the learning and ex-

\footnotetext{
Received Jan. 12, 2015; revised April 19, 2015; accepted April 24, 2015.

Author contributions: A.F. and H.E. designed research; A.F., R.J.P., B.P., and C.E.M. performed research; A.F. and S.M. analyzed data; A.F. and H.E. wrote the paper.

This research was supported by National Institute of Mental Health (NIMH) Grant MH094263. We thank Lara Rangel and Jon Rueckemann for helpful comments on analysis, and Edward Meagher for his effort in the coding of the video files.

The authors declare no competing financial interests.

Correspondence should be addressed to Howard Eichenbaum, Center for Memory and Brain, Boston University, 2 Cummington Street, Boston 02215, MA. E-mail: hbe@bu.edu.

DOI:10.1523/JNEUROSCI.0134-15.2015

Copyright $\odot 2015$ the authors $\quad 0270-6474 / 15 / 358333-12 \$ 15.00 / 0$
}

pression of memories where the context defines the outcomes of events.

Of particular relevance to the current study, where context guides the retrieval of appropriate reward associations of events, many previous studies have implicated the orbitofrontal subdivision of prefrontal cortex (OFC) in representing expected rewards or aversive outcomes (Rolls et al., 1996; Tremblay and Schultz, 2000; Furuyashiki and Gallagher, 2007; Wallis, 2007; Mainen and Kepecs, 2009; Schoenbaum et al., 2009; Sul et al., 2010; Rudebeck and Murray, 2014). Several studies have shown that OFC damage results in an inability to change behavior when reinforcement contingencies are altered, as well as a loss of the ability to distinguish currently relevant from irrelevant memories (Schnider and Ptak, 1999; McAlonan and Brown, 2003; GhodsSharifi et al., 2008; Young and Shapiro, 2009). Moreover, OFC neuronal firing patterns change dynamically as rats learn new reward outcomes associated with specific events (Schoenbaum et al., 1999; Young and Shapiro, 2011). These observations strongly suggest a role of the OFC in behavioral flexibility, possibly by way of forming representations of outcome expectancies to inform which cues and behaviors lead to reward (Ostlund and Balleine, 2007; Schoenbaum et al., 2009, 2011; Padoa-Schioppa and Cai, 2011). We specifically tested the hypothesis that OFC ensembles contain a mapping of contextual cues and specific stimuli associated with behavioral responses and the values of consequent reinforcement outcomes (Wilson et al., 2014). Here we recorded simultaneously from multiple OFC neurons as rats learned and subsequently retrieved memories about which of two objects was rewarded in each of two environmental contexts. Results from single-neuron and neural population analyses revealed the development of a systematic organized representation, or schema, of 
task-relevant dimensions observed during object sampling, as well as strong coding of the distinct environmental contexts that predict the different object-reward associations during context exploration before object sampling.

\section{Materials and Methods}

Animals. Four male Long-Evans rats (Charles River), weighing between 225 and $250 \mathrm{~g}$ at the start of the experiment, were used in the study. All animals were singly housed and maintained on a $12 \mathrm{~h} \mathrm{light/dark} \mathrm{cycle} \mathrm{(lights} \mathrm{on} \mathrm{8:00} \mathrm{A.M.}$ to 8:00 P.M.). Behavioral training and testing were conducted during the light phase. Animals were kept at $85 \%$ of their ad libitum feeding body weight and had ad libitum access to water in the home cage. Procedures were conducted according to the requirements set by the National Institutes of Health and Boston University Institutional Animal Care and Use Committee.

Materials and apparatus. Behavioral training and testing were performed in a custom-made behavioral environment $(160 \mathrm{~cm}$ length $\times 60$ $\mathrm{cm}$ width $\times 40 \mathrm{~cm}$ height) that consisted of two separate chambers (contexts) connected via an alleyway, and access to each context was controlled by dividers at each entry. Each context had a distinct texture on the floor and walls. The objects consisted of ceramic pots $(10 \mathrm{~cm}$ high with an internal diameter of $9 \mathrm{~cm}$ ), and each pot was filled with a distinct digging medium (e.g., purple beads, multicolor gum elastic squares, shredded paper, small pebbles, bits of yarn, and pieces of soda straws). Rewards were one-quarter of a Froot Loop (Kellogg's) buried in the pot assigned as rewarded. To prevent the animal from being guided by the smell of the Froot Loop, the media were sprinkled with crushed Froot Loops.

Behavioral protocol. OFC neuronal activity was monitored in rats during the retrieval of memories where two distinct environmental contexts ( 1 and 2) predict different reward expectations of behavioral responses to two distinct objects (A and B) presented in either of two positions within each context. When presented at either position in Context 1 , choosing Object A was rewarded and choosing Object B was not rewarded, whereas in Context 2, choosing Object B was rewarded and Object A was not (Fig. 1).

Animals were trained in successive stages. Initially, rats were trained to dig for a reward (one Froot Loop) buried in a pot filled with unscented sand. Once the animals had learned to dig reliably to retrieve the reward, they were trained on a simple odor discrimination task in the home cage. Two pots filled with sand and scented with two distinct odors (cinnamon and cumin household blends) were placed at opposite corners of the home cage, and on each trial the location of each odor was pseudorandomly determined. Cinnamon was always rewarded, and cumin was never rewarded, and the animal had to learn which odor was associated with reward. Once the animal reached a criterion of $80 \%$ correct across 20 consecutive trials, the animal was habituated to the subsequently used testing apparatus by being allowed to explore for $30 \mathrm{~min}$ while retrieving scattered Froot Loops.

During training, each session consisted of 90 trials, 45 trials in each context. Each trial consisted of a context exploration period that lasted $10 \mathrm{~s}$, followed by an object-sampling period. During the context exploration period, the animal was allowed to enter and explore one of the contexts in the absence of the objects (Fig. 1). At the end of this period, a divider was positioned within the context area to keep the animal away from the pot positions, while Objects A and B were placed in the corners opposite the entry to the context. Once the pots were in place, the divider was removed, allowing the rat to approach the pots from within the context. In each problem, reward and nonreward associations were arbitrarily assigned to object pairs, and one-quarter of a Froot Loop was buried in the pot accordingly on each trial. If the animal first approached the rewarded object, it could dig in the pot to retrieve the food reward. If, however, the nonrewarded object was approached first, the animal had to refrain from digging in the pot, and then sample the other object to retrieve a reward. If the animal did not approach a pot within $20 \mathrm{~s}$, the trial was terminated, and the animal was shuffled to the other context. Object position within each context was pseudorandomized, and no object occurred in the same location for more than two consecutive trials. After most trials, the animal moved into the opposite context via the alleyway. However, on nine trials for each context, the animal remained in the same context for an additional trial to prevent a strict alternation strategy. On these trials, the animal was moved away from the objects using a divider then, after a context exploration period during which the object positions were changed on half of these trials, the divider was removed, allowing access to the objects. In the initial problem acquired before implantation of electrodes, animals were trained to reach a performance criterion of $80 \%$ correct within a session, and they required three to six sessions to reach this criterion. After microdrive implant, animals were retrained on the same problem, and presented with four to nine new object-context association problems that involved novel digging mediums and contextual cues. Following the initial learning session in which criterion performance was obtained on a new problem, animals were presented with the same problem in an additional post-training session, which provided the data for this study.

Surgery. Anesthesia was induced by the inhalation of 5\% isoflurane (Webster Veterinary Supply) in oxygen and was maintained at 2-2.5\% throughout surgery. Animals were given the analgesic Buprenex (Buprenorphine hydrochloride, $0.03 \mathrm{mg} / \mathrm{kg}$, i.m.; Reckitt Benckiser Healthcare Ltd.) and placed in a stereotaxic frame (KOPF), where an incision was made along the midline to expose the skull. Animals were implanted with microdrives that contained 24 independently drivable tetrodes aimed at the ventral orbital (VO)-lateral orbital (LO) regions at coordinates obtained from Paxinos and Watson (2007) (anteroposterior, 4.5 $\mathrm{mm}$; mediolateral, $\pm 1.6 \mathrm{~mm}$ ). Each tetrode consisted of four $12 \mu \mathrm{m} \mathrm{RO}$ 800 wire (Sandvik Kanthal HP Reid Precision Fine Tetrode Wire, Sandvik) that was gold plated to reduce impedance to between 180 and $220 \mathrm{k} \Omega$ at $1 \mathrm{kHz}$. At the end of surgery, each tetrode was lowered $\sim 2.5 \mathrm{~mm}$ into tissue. Postsurgery, animals were given Buprenex $(0.03 \mathrm{mg} / \mathrm{kg}$, i.m.), and Cefazolin (330 mg/ml, i.m.; West-Ward Pharmaceuticals) Animals were rested for 1 week before behavioral testing resumed.

Neural recordings. Electrical recordings were made using a 96-channel Multichannel Acquisition Processor (Plexon). Each channel was ampli- 


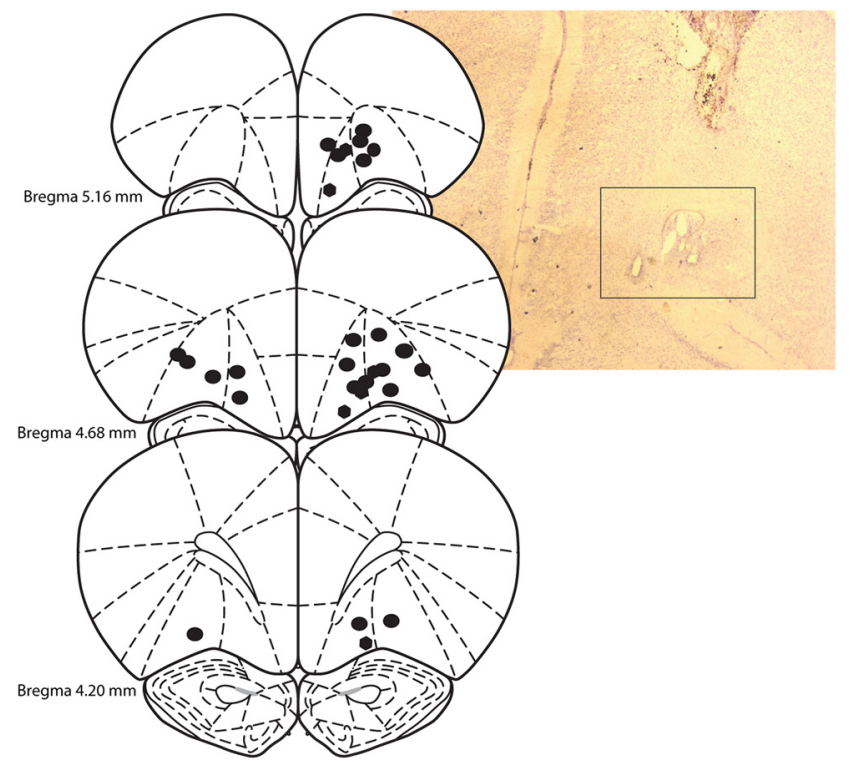

Figure 2. Reconstruction of recording sites in V0 and $\mathrm{L} 0$ cortices at $\sim 4.20,4.68$, and 5.16 $\mathrm{mm}$ anterior to bregma in animals recorded from coordinates taken from Paxinos and Watson (2007). Black dots indicate the site of the tetrode tip after recording had ended. Photomicrograph depicts burn marks from tetrode tips.

fied and bandpass filtered for both single-unit activity $(154 \mathrm{~Hz}-8.8 \mathrm{kHz})$ and local field potentials $(1.5 \mathrm{~Hz}-400 \mathrm{kHz})$. Spike channels were referenced to another electrode in the same region to remove movementrelated noise. Action potentials were detected by threshold crossing and digitized at $40 \mathrm{kHz}$. Each recording of units and local field potentials was made using SortClient and Cineplex Studio for video recording (Plexon). Single units were isolated using Offline Sorter (Plexon), and behavioral events were time stamped using Cineplex Editor (Plexon). All data analysis was performed using custom scripts for MATLAB (MathWorks). To reduce the likelihood of recording from the same neuron across multiple sessions, tetrodes were lowered before each testing session $(\sim 0.018 \mathrm{~mm}$ or more), and the amount to which a tetrode was lowered was based on a visual inspection of the identified units.

ANOVA on single neuron firing patterns. To determine the selectivity of single-neuron activity to object, position, and context during object sampling, firing rates were calculated for each sampling event beginning when the nose of the animal reached the edge of a pot and ended when the rat began to dig or withdrew on all events in which a correct response was subsequently made. Statistical differences in mean firing rate among objects, positions, and contexts for each neuron were determined using a three-factor nested ANOVA with context (two levels), position (two levels nested within each of the two contexts), and object (two levels) as factors. To determine the selectivity of single-neuron activity for different context exploration events, firing rates were calculated for each context exploration event beginning when the animal entered a context and for the subsequent $5 \mathrm{~s}$. Then statistical differences in mean firing rate between explorations of the two distinct contexts were determined using a one-way ANOVA with context as a factor (two levels). A $p$ value of 0.05 was used to determine significance.

Representational similarity analysis of neural ensemble activity patterns. Firing rates for each neuron obtained in the single-neuron analyses described above were $z$-transformed across all object-sampling events, then the mean $z$-transformed firing rate associated with each type of objectsampling event (e.g., Object A in Position 1 within Context 1) and for exploration periods in both contexts were calculated to compose a set of population vectors for each recording session. Neural ensemble firing patterns were explored using a representational similarity analysis (RSA) to determine the extent to which multiple task dimensions were encoded by OFC (Kriegeskorte et al., 2008; McKenzie et al., 2014). To measure the similarities between ensemble representations of different types of object-sampling events, pairwise Pearson product-moment correlations were calculated between population vectors taken for events that were the same or different in each of the three dimensions (object, position, and context), thus composing six basic comparisons composed of correlations between population vectors for object-sampling events involving the same or different objects in the same or different positions and the same or different contexts (see Fig. 4A). The magnitude of the correlation coefficient for each comparison reflects the representational similarity of events being compared. A positive correlation coefficient indicates representational similarity in population coding of the dimension tested; zero correlation indicates independence of representations for that dimension; and a negative correlation coefficient (i.e., anticorrelation) suggests strong pattern separation of that dimension by the neuronal ensemble.

First, to measure the extent to which identical events were coded similarly, population vectors for odd-numbered events were correlated against those for even-numbered events for each of the two objects within each of the two positions in each of the two contexts. The mean of those eight correlation coefficients was used to measure the ensemble similarity for identical events within each of the 33 recording sessions (see Fig. 4A, Bar 1). For all other comparisons, population vectors for object-sampling events of each type were correlated with those for a different type of event. To measure the similarity of ensemble representations for different objects sampled at the same position, population vectors for odd-numbered events for one object were correlated against even-numbered events for the other object, and vice versa (evennumbered events for the first object against odd-numbered events for the second object), to compose eight total correlations, and the mean of those correlation coefficients was used to measure the representational similarity for different objects (holding position and context constant) in each session (see Fig. 4A, Bar 2). The same approach was used to measure the representational similarities for the same or different objects at different positions within a context, including the separation of odd- and even-numbered events to ensure that similar amounts of data were used in all analyses. To assess the similarity of ensemble representations for the same object between positions, population vectors for sampling events involving an object in one position were correlated with those for the same object in the other position within the same context (see Fig. 4, Bar 3 ) or with that for the other object in the other position within the same context (see Fig. 4, Bar 4), again comparing odd-numbered against evennumbered events and vice versa. To assess the similarity of ensemble representations of objects between contexts, population vectors for oddnumbered and even-numbered events for the same object (see Fig. 4, Bar 5) or different objects (see Fig. 4, Bar 6) at positions between contexts were similarly correlated.

We also measured the extent to which ensembles represented context exploration events within the two environments as similar or distinct. To measure the similarity of ensemble representations of events involving exploration of the same context, population vectors for odd-numbered versus even-numbered exploration events within each context were calculated separately, and then the population vectors for odd-numbered events were correlated with those for even-numbered events, and vice versa, for each context, and the mean of those correlation coefficients was used to measure the extent to which contexts were represented by OFC ensembles. To measure the similarity of ensemble representations of exploration events between contexts, population vectors for oddnumbered events in one context were correlated with even-numbered events in the other context, and vice versa, and the mean of those correlation coefficients was used to measure the extent to which events involving the exploration of meaningfully different contexts were represented similarly (see Fig. 7B).

Bootstrap analysis. For each neuron, firing rates were shuffled between sampling event identities (e.g., sampling of Object A in Position 1 in Context 1 is an event identity). For each shuffle, the mean firing rate of each neuron for each possible event identity (eight identities in total; two objects and four positions) was recalculated. This reassignment of firing rates between event identities was performed for each neuron in the population vector, and where each neuron in the vector now had a new mean firing rate for each event identity. Correlations were then performed on population vectors for each of the comparisons shown in 
Figure $4 A$ to obtain a correlation coefficient for each comparison. This procedure was repeated 10,000 times, and the observed correlation coefficient obtained for each comparison before shuffling the firing rates was compared with a distribution of correlation coefficients from the shuffled dataset. To determine whether differences obtained between conditions of interest (see Fig. $4 A$, between bars) were greater than that expected by chance, we first calculated the difference between the mean correlation coefficient $r$ for one condition (e.g., Bar 1, mean $r=0.36$ ) and the mean correlation coefficient $r$ for the other condition (e.g., Bar 2, mean $r=-0.19$ ) by subtracting the value of $r$ for one condition from the value of $r$ obtained for the other condition $\left(r_{\text {diff }}\right)$. We then compared the $r_{\text {diff }}$ obtained against a distribution of 10,000 $r_{\text {diff }}$ values for that same comparison after the firing rates had been shuffled between event identities. To account for multiple comparisons, in analyses of objectsampling events, a Bonferroni correction was applied by dividing $p=$ 0.05 by the number of conditions (see Fig. $4 A$, six comparisons), which yielded a statistical threshold of $p=0.05 / 6=0.008$. Statistical significance at $p<0.008$ was reached when the observed correlation was $>9920$ of the correlations for shuffled datasets. For context exploration events, $p=0.05$ was divided by two conditions (see Fig. $7 B$, two comparisons), which yielded a statistical threshold of $p=0.05 / 2=0.025$ ). Statistical significance at $p<0.025$ was reached when the observed correlation was $>9700$ of the correlations for shuffled datasets.

Dendrogram analysis of the organization of ensemble representations. To explore the organization of ensemble representations for the eight types of object-sampling events (i.e., conjunctions of two objects in two positions within two contexts), we generated composite ensemble representations for each event type using the firing rates of all of the 394 neurons collected across 33 sessions. The composite population vector for each type of event was calculated as follows: for each neuron, the number of spikes observed was divided by the sampling duration on each event, then these firing rates were standardized into $z$-scores using the mean and SD of firing rates across events, then the mean $z$-score firing rates across events was calculated. The relationships among the composite population vectors for the eight types of events were then assessed using an agglomerative hierarchical clustering algorithm (MATLAB R2013b function "linkage"). The agglomerative hierarchical clustering algorithm takes the unweighted average distance between pairs of the eight vectors, where the Pearson's correlation coefficient was used as the distance metric. Of the eight vectors, the two that were nearest were combined, then calculations of distances between the revised set of vectors was repeated, and the nearest two vectors were combined; this process was repeated iteratively until only two combinations remained (Fig. 4B). The height of each line in the dendrogram represents the similarity (mean $r$ value) between the event types being connected.

Dynamics of ensemble coding during object sampling. To determine the time course of ensemble representations of position and objects and their associated values during object sampling, we used a $d^{\prime}$ metric to compare the average correlations for the same [e.g., Object A (or B) to itself] and different comparisons (e.g., Object A to Object B) for each of these dimensions (see Fig. 8). Only the first object-sampling event on each trial and only events that were longer than $500 \mathrm{~ms}$ were included in the analysis. Correlation analysis was performed for sequential $200 \mathrm{~ms}$ time bins starting after the approach to the pot began and $-3.4 \mathrm{~s}$ and ending at $+3.4 \mathrm{~s}$ relative to the onset of object sampling. The $d^{\prime}$ metric estimated the separation between the mean correlations for the same and different conditions, and applied the combined variance of each same and different condition to obtain the distance between means of the conditions compared. Thus, the greater $d^{\prime}$ is from 0 , the greater the representational separation for comparisons made. The $d^{\prime}$ values were obtained using the following equation, where mean ${ }_{1}$ and $\mathrm{SD}_{1}^{2}$ reflect the correlation mean and variance, respectively, for one condition, and mean $_{2}$ and $\mathrm{SD}_{2}^{2}$ reflects the correlation mean and variance, respectively, for the second condition. To account for multiple comparisons, we applied Bonferroni correction by dividing $p=0.05$ by the number of bins ( 34 bins), which yielded a $p$ value of $0.05 / 34=0.0015$ for statistical significance to be reached:
Table 1. Encoding of task dimensions by single cells

\begin{tabular}{lccccr}
\hline & \multicolumn{2}{l}{ Inclusive } & & & Exclusive \\
\cline { 2 - 3 } \cline { 6 - 6 } Dimensions & Count & $\%$ & & Count & $\%$ \\
\hline Context & 60 & 15 & 18 & 5 \\
Position & 54 & 14 & 13 & 3 \\
Object & 62 & 16 & 13 & 3 \\
Object $\times$ context & 149 & 38 & 76 & 19 \\
Object $\times$ position & 48 & 12 & 5 & 12 \\
Context exploration & 55 & 14 & 47 & 12 \\
\hline
\end{tabular}

Encoding of task dimensions by single neurons. Number and percentage of neurons (from 394 neurons recorded) that met statistical criteria for differentiating task dimensions during object sampling, and during context exploration. In the "Inclusive" column, neurons may be counted in more than one single dimension or interaction. For context exploration, inclusive involves neurons that also differentiated task dimensions during object sampling Each value in the "Exclusive" column had significant selectivity for only the particular dimension or interaction specified.

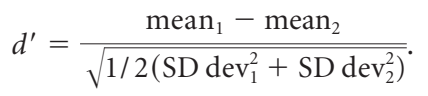

Histology. After completion of behavioral testing, tetrode placements were confirmed by creating a lesion at the tetrode tip by passing a $40 \mu \mathrm{A}$ current until the connection was severed on each tetrode wire. Animals were subsequently overdosed with systemic injection of Euthasol (Virbac $\mathrm{AH})$ and were perfused intracardially with $0.9 \%$ saline followed by $10 \%$ formalin phosphate (VWR). Brains were removed and placed in a $20 \%$ sucrose solution until processed. Using a cryostat (CM 3050s, Leica Biosystems), brains were cut into $35 \mu \mathrm{m}$ coronal sections and mounted onto presubbed glass slides, and stained with cresyl violet to determine the location of tetrode tip lesions. Tetrode tip lesions were confirmed to be localized within either ventral or lateral orbitofrontal cortex (Fig. 2) using the stereotaxic atlas of Paxinos and Watson (2007). We were not able to determine which exact tetrode belonged to ventral or lateral OFC.

\section{Results}

Across four animals, a total of 394 single neurons in the ventral and lateral orbital cortices (Fig. 2) were recorded over 33 sessions that followed the initial learning of each problem. Rat 1 contributed 69 neurons ( 6 sessions), Rat 2 contributed 37 neurons (9 sessions), Rat 3 contributed 91 neurons (12 sessions), and Rat 4 contributed 197 neurons (6 sessions). During these sessions, animals performed the task with high accuracy (mean, 95\% correct; SEM, $0.7 \%$ correct; range, $92-100 \%$ correct). Analyses separately explored neural activity patterns during the period of object sampling leading to the behavioral response and during the preceding period of context exploration when rats entered and explored one of the contexts before being presented with the objects. Our analysis of these trial periods characterized both single-neuron firing patterns and the firing patterns of simultaneously recorded neural ensembles in OFC.

\section{OFC neurons encode each dimension of context-guided object-reward associations}

OFC neurons encoded the full range of dimensions that characterize each object-sampling event. Overall, 194 of 394 isolated neurons (49\%) encoded task-related dimensions of objectsampling events that were followed by the correct behavioral response to the presented stimulus. Substantial proportions (14$16 \%$ ) of the neurons fired differentially associated with the object, position, or context dimensions (main effects), although few of these fired exclusively associated with one dimension (Table 1). A similar proportion of neurons encoded objects in specific positions within a context (significant object $\times$ position interaction). Most notably, $38 \%$ of neurons distinguished combinations of objects and the context in which they were sampled (significant object $\times$ context interaction), and the firing pattern of $>50 \%$ of 
Cell 1
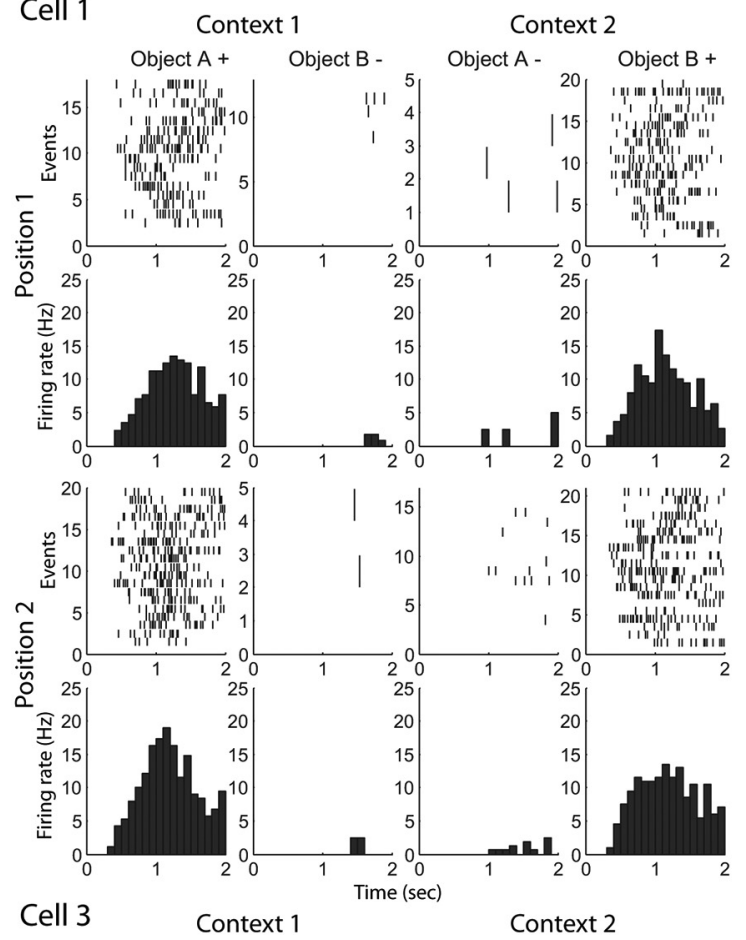

Cell 2
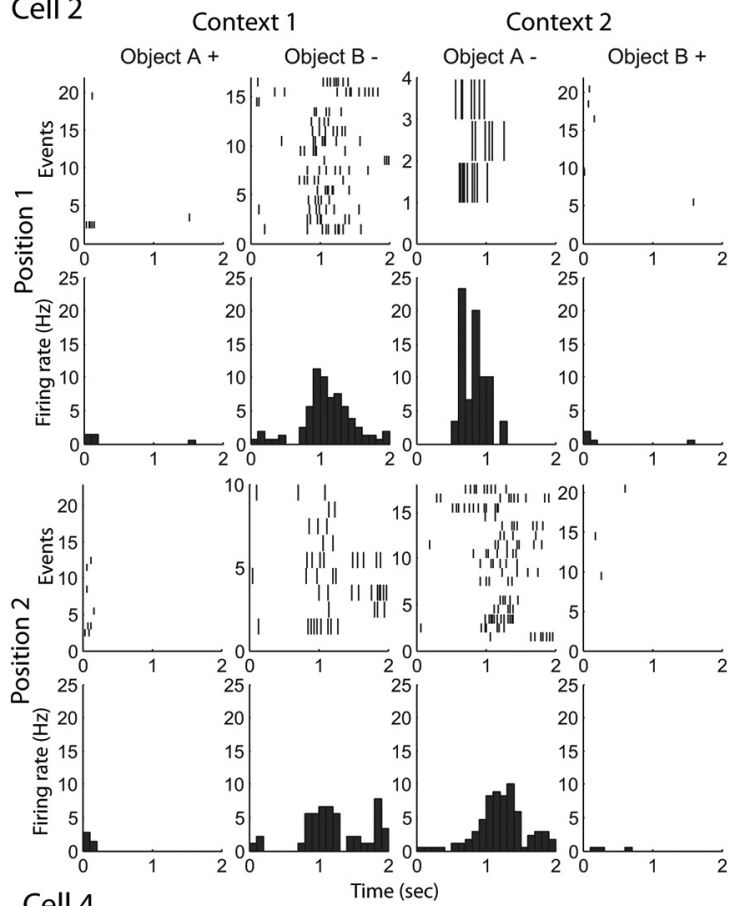

Cell 4
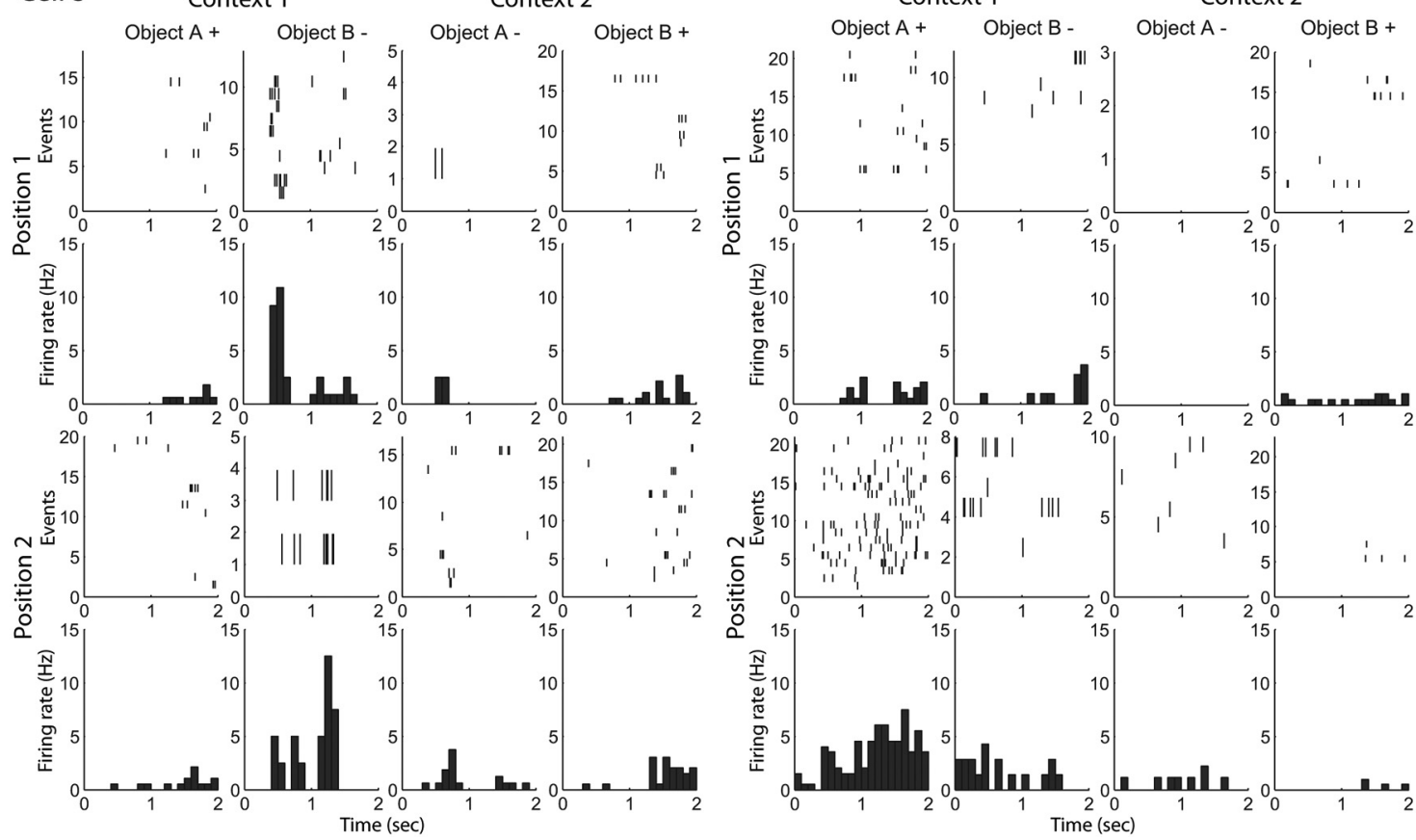

Figure 3. Examples of single-cell selectivity to different task dimensions during the sampling of objects in each context. Rasters and perievent histograms depict activity patterns of example neurons during the sampling of each object ( $A$ or B) at each position (1 or 2) within each context (1 or 2). Rewarded ( + ), unrewarded ( - ). Rasters show spikes for each object-sampling event. Time 0 indicates the onset of object sampling. The histogram represents firing rate in $100 \mathrm{~ms}$ time bins.

these neurons was characterized by equivalent activation during the sampling of different objects in the opposing contexts, thus reflecting the common reward value of these events. However, reward associations did not fully account for differences in the activity across object-context combinations in many other cells. Figure 3 shows the firing patterns of four example neurons. Cell 1 fired strongly and at equivalent rates during the object-sampling events associated with reward (Object A in Context 1 and Object $B$ in Context 2) and not during object sampling that was not associated with reward (object $\times$ context interaction: $F_{(1,103)}=$
202.84, $p=0.0001$ ), and did not fire differentially associated with objects or contexts alone (main effects) or other dimensions. Cell 2 fired during object-sampling events associated with nonreward (Object B in Context 1 and Object A in Context 2) and did not fire during object-sampling events associated with reward (object $X$ context interaction: $\left.F_{(1,121)}=9.6, p=0.002\right)$; the activity of this cell also differed across contexts (main effect: $F_{(1,121)}=18.59, p=$ 0.0001 ) and between objects (main effect: $F_{(1,121)}=8.92, p=$ 0.003 ), indicating that reward association was not the only dimension encoded. Cell 3 fired selectively to the nonrewarded 
Object B in Context 1 (main effect object: $F_{(1,103)}=20.62, p=$ 0.0001 ) but was not object selective in Context 2 (object $\times$ context interaction: $F_{(1,103)}=23.85, p=0.0001$; main effect context: $\left.F_{(1,103)}=16.9, p=0.0001\right)$. Finally, Cell 4 fired selectively during object sampling at Position 2 within Context 1 compared with the other positions (main effect position: $F_{(1,101)}=14.21, p=$ 0.0001 ), and fired selectivity within Context 1 , which included its preferred position, compared with Context 2 (main effect context: $F_{(1,101)}=5.01, p=0.03$ ). Thus, in addition to reward associating firing, many individual cells also differentiated the objects, positions, and contexts. More generally, while some cells exclusively encoded only one of the task dimensions, most exhibited mixed selectivity and overall, ensembles encoded the full range of task dimensions, consistent with high-dimensional ensemble representations that can support a broad domain of cognitive functions (Rigotti et al., 2013). To examine whether there were differences in firing patterns on trials that were performed sequentially within the context, we extracted the trials in which sampling events occurred within the same context for two successive trials (nine repeat trials per context in a session) and performed a one-way ANOVA on the firing rates, combined across all neurons, for Object $\mathrm{A}$ and Object $\mathrm{B}$ during the first and second (repeat) trial. We observed no significant differences in firing rates during object sampling between the two successive trials $\left(F_{(3,131)}=0.40, p=0.75\right)$, suggesting that a repeat trial within a context did not alter spiking activity during object sampling.

\section{OFC neuronal ensembles represent multiple task dimensions during object sampling}

To explore how neural populations represented the task dimensions, we used an RSA in which $z$-scored firing rates of a set of simultaneously recorded neurons were composed into population vectors (lists of mean firing rates) for each event identity and then the population vector for an event identity was crosscorrelated against that of other event identities (see Materials and Methods). These correlations provided a measure of the similarity of population representations associated with each task dimension. The RSA analysis was applied to population vectors composed of data from ensembles of 3-50 simultaneously monitored neurons (mean, 14 neurons). The analyses revealed striking ensemble representations of each of the task-relevant dimensions during object sampling (Fig. 4A).

\section{Value coding}

As a first approach to exploring value coding, based on the observation of a large proportion of single OFC neurons whose firing patterns differed depending on the associated reward value (rewarded or nonrewarded), we examined comparisons in which the position and context dimensions were held constant while the objects that predicted the same or different reward value varied. We observed a strong positive correlation between population vectors of object-sampling events that involved the same object in the same position, and therefore the same reward value (Bar 1: mean $r=0.36$, SEM $=0.04$ ), and a pronounced negative correlation between population vectors for events that involved different objects in the same position and therefore opposite reward value (Bar 2: mean $r=-0.19$, SEM $=0.04$; Bar 1 vs Bar 2, $p<$ $0.0001)$. The same pattern emerged when we compared population vectors for object-sampling events that involved the same object, and therefore same reward value, in different positions in the same context (Bar 3: mean $r=0.22$, SEM $=0.04$ ) versus different objects in different positions and therefore different reward values (Bar 4: mean $r=-0.27$, SEM $=0.03$; Bar 3 vs Bar 4,

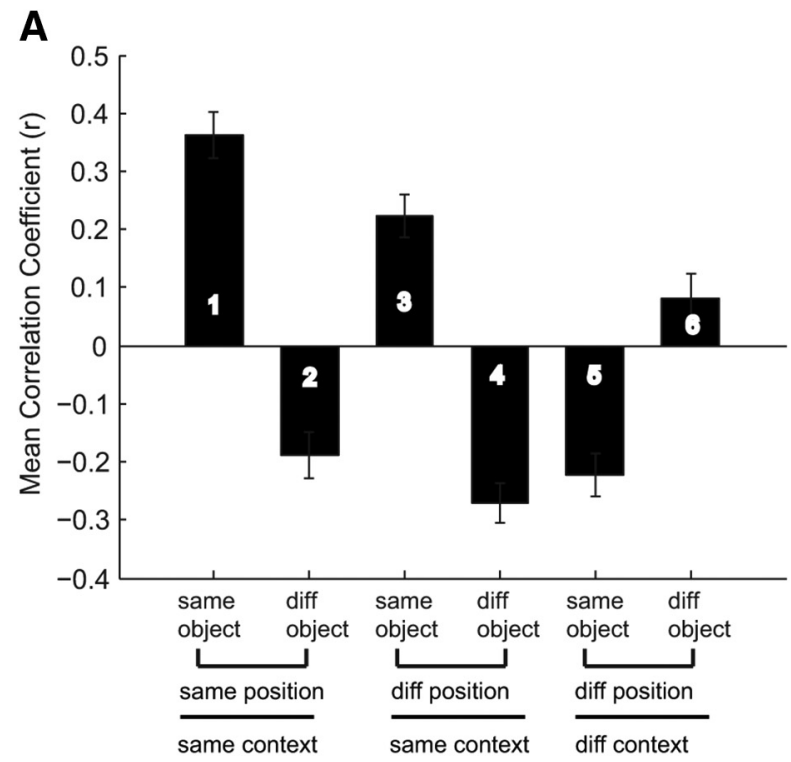

B

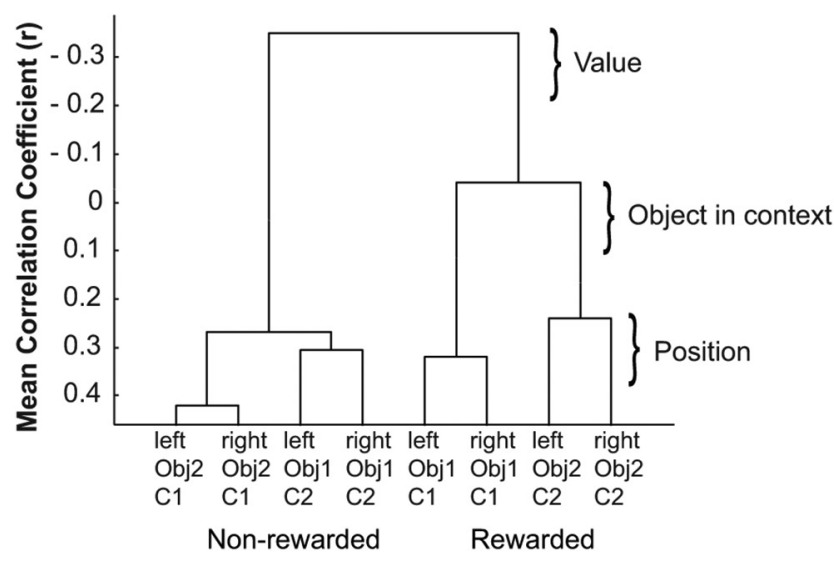

Figure 4. Population analyses. $\boldsymbol{A}$, Mean Pearson correlation coefficient $(r)$ of population vectors (SEM across sessions). Diff, Different. "Same object" and "different object" refer to comparisons of types of events that involve the same or different object identities, respectively. Same position and different position refers to comparisons of types of events that involve the same or different object positions within a context, respectively. "Same context" and "different context" refers to comparisons of types of events that involve the same or different contexts, respectively. Note that events that are compared across contexts necessarily involve different positions; these are considered different context comparisons. The specific comparisons were as follows: 1, Same object in the same position and context (e.g., Object A events versus other Object A events in Position 1 and (ontext 1); 2, different objects in the same position and context (e.g., Object A vs B in Position 1 and Context 1); 3, same objects in different positions in the same context (e.g., Object A in Position 1 vs Object A in Position 2 in Context 1); 4, different objects in different positions in the same context (e.g., Object $A$ in position 1 vs Object B in Position 2 in Context 1); 5, Same objects in different positions within different contexts (e.g., Object A in Context 1 vs Object $A$ in Context 2); and 6, different objects in different position and in different contexts (e.g., Object $A$ in Context 1 vs 0 bject B in context 2). $\boldsymbol{B}$, Dendrogram shows the organization of event dimensions in OFC ensembles during object sampling as a function of similarities of population vectors along different dimensions of events. Note the strong dissimilarity (anticorrelation) between events with opposite associated reward values, the independence of representations for different rewarded objects in different contexts, the similarity of different nonrewarded objects in different contexts, and the stronger similarity of the same events in different positions. C1, Context 1; C2, context 2; 0bj, object; left, left position; right, right position.

$p<0.0001$ ), as well as in the comparison of population vectors for object-sampling events that involved the same object in different contexts, and therefore opposite reward value (Bar 5: mean $r=-0.22, \mathrm{SEM}=0.04)$ versus different objects in different 
contexts and therefore the same reward value (Bar 6: mean $r=$ 0.08 , SEM $=0.04$; Bar 5 vs Bar $6, p<0.0001$ ).

We also performed additional confirmatory analyses combining different types of comparisons that shared the same reward values across variations in other dimensions. In this analysis, we measured the overall representational similarities of ensemble firing patterns during object-sampling events associated with the same reward value by combining the comparisons for all types of events with the same reward outcome (Fig. 4A, compare Bars 1, 3, and 6), and found a very high strength-of-value coding (mean $r=$ 0.25 ; SEM $=0.03$ ). Conversely, when we combined the correlations that compare all types of events with opposite reward value (Fig. 4A, compare Bars 2, 4, and 5), we found a strong anticorrelation, indicating striking differentiation of opposite reward value by OFC ensembles (mean $r=-0.24$; SEM $=0.02$ ). We next compared the combined correlations for all types of events with same reward value (Bars 1, 3, and 6) against the combined correlations for all types of events with different reward values (Bars 2, 4, and 5), and confirmed the difference between representational similarities of population coding for object-sampling events associated with the same versus the opposite reward value $(p<0.0001)$. Together, this pattern of representational similarities suggests that OFC ensembles have similar representations for events associated with the same reward value, even those involving different objects, locations, or contexts, albeit at different strengths associated with these other dimensions. Conversely, representations of events associated with opposite reward value are strongly dissimilar.

Object in context coding

Guided by the findings on single-cell analyses that revealed a substantial proportion of neurons that were differentially activated during sampling of the same object in distinct contexts (Fig. 3 ), we next explored the representation of distinct object-context (i.e., different object and different context) combinations that shared the same reward outcomes. This was accomplished by comparing correlations of events that involved the same object at different positions in the same context, and therefore having the same value (Bar 3: e.g., Object A vs Object A at different positions within Context 1, both rewarded) versus that for events that involved different objects in different contexts, and therefore had the same value (Bar 6: e.g., Object A in Context 1 vs Object B in Context 2, both rewarded). This correlation for events with the same object-context combinations was greater than that for the correlation between population vectors for events that involved different objects and different contexts with the same reward value (Bar 3 vs Bar $6, p<0.0001$ ), indicating that object-context coding occurred beyond representation of the reward outcomes of different events per se.

The coding of object-context combinations differed for the subset of object-sampling events associated with reward and for the subset of events associated with nonreward. Population vectors for object-sampling events that involved the same objectcontext combination with an expectation of reward were positively correlated (Fig. 4A, Bar 3, for rewarded events only; mean $r=0.20$, SEM $=0.03$ ). This correlation between population vectors for events with the same object-context combinations and reward association was greater than that for different object-context combinations associated with reward (Bar 6 for rewarded events only: mean $r=-0.007$, SEM $=0.05$; Bar 3 vs Bar 6 for rewarded events only, $p<0.001)$. Also, population vectors for object-sampling events that involved the same object-context combination with an expectation of nonreward were positively corre- lated (Bar 3 for nonrewarded events only: mean $r=0.25$, SEM $=$ 0.04). However, correlation between population vectors for events with the same object-context combinations for nonrewarded events was not significantly greater than that for opposite nonrewarded object-context combinations (Bar 6 for nonrewarded events only: mean $r=0.18$, SEM $=0.05$; Bar 3 vs Bar 6 for nonrewarded events only, $p=0.14$, n.s.). Finally, representations for nonrewarded events in different contexts were more similar than those for rewarded events, and the difference in representational similarity between rewarded and nonrewarded events was significant $(p<0.001)$.

\section{Position coding}

To assess position coding, we compared population vectors for object-sampling events in which position differed while the reward value and object-context dimensions were held constant. Position coding was evident in stronger similarity of population vectors for object-sampling events that involved the same object in the same position (Fig. 4A, Bar 1) versus events with the same object in the alternate position within a context (Fig. 4A, Bar 3), where both comparisons were associated with the same object-context combination and reward value while differing only in position (Fig. $4 A$, Bar 1 vs Bar 3; $p<0.001)$. Also, the correlation between population vectors for events with different objects in the same position (and therefore different values; Fig. 4A, Bar 2) was less negative than that for events with different objects in the alternate position within the same context (Fig. 4A, Bar 4), but this difference was only marginally significant in the Bonferroni-corrected test (Fig. 4A, Bar 2 vs $\operatorname{Bar} 4 ; p=0.03)$.

\section{The same representational pattern is apparent when small neuronal ensembles are eliminated}

For the analysis described above, all ensembles that were composed of at least three neurons were included. To determine whether the activity patterns of very small ensembles might have outsized influence on the overall results, we repeated the analysis including only ensembles of at least 10 neurons, thus excluding 14 of 33 sessions and leaving out the data from Rat 2 where all ensembles involved $<10$ neurons. However, even with this reduced dataset, the population firing patterns were strikingly similar to those including the full dataset (compare Fig. 5A, Fig. 4A). To assess whether population representations were similar after excluding ensembles consisting of $<10$ neurons, we made three comparisons (Fig. 5A). We compared all correlations between population vectors for object-sampling events that involved the same value (Fig. 5A, combined correlations of Bars, 1, 3, and 6) versus those for all events that involved different values (Fig. 5A, combined correlations of Bars 2, 4, and 5). Population representations continued to show strong value coding, similar to the overall combined analysis $(p<0.001)$. Additionally, we compared correlations between population vectors of sampling events that involved the same object in the same position within a context with those for events that involved the same object between positions within each context (Fig. 5A, Bar 1 vs Bar 3), and population representations continued to show position coding $(p<0.008)$. Finally, we compared the correlations of population vectors of sampling events that involved the same object between positions within a context (therefore holding reward value constant) versus those for events that involved different objects in different contexts (thus also holding reward value constant; Fig. $5 \mathrm{~A}$, Bar 3 vs Bar 6), and population representations continued to show object-in-context coding ( $p<0.008$; Fig. $5 A)$. 
A

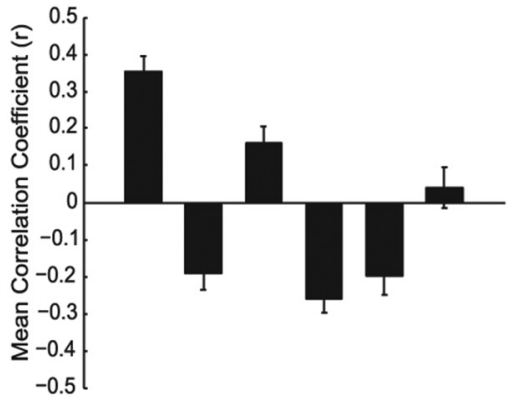

B

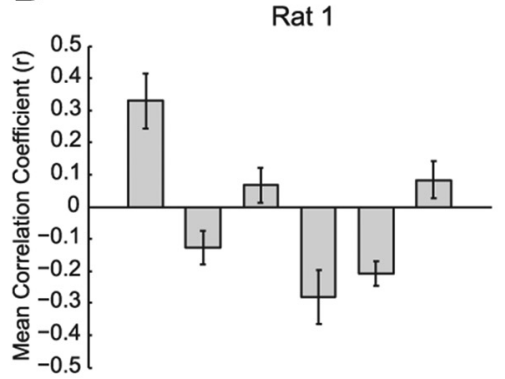

Rat 2

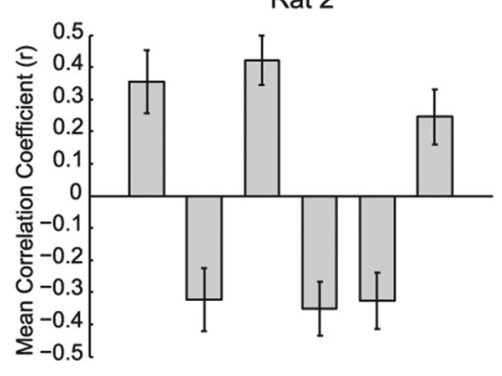

Rat 3

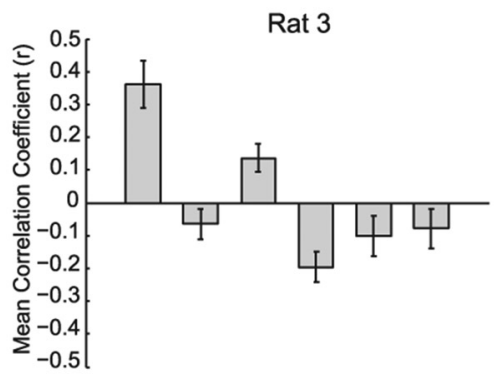

Rat 4

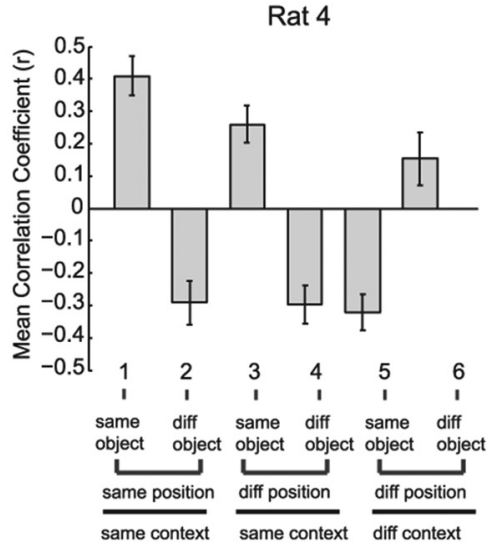

Figure 5. $\quad A, \mathrm{RSA}$ for group data from ensembles that consisted of at least 10 neurons. $B, \mathrm{RSAs}$ for each animal. Diff, Different. See description of conditions in Figure $4 A$.
A Rewarded object approached 1st $\mathbf{B}$ Rewarded object approached 2nd
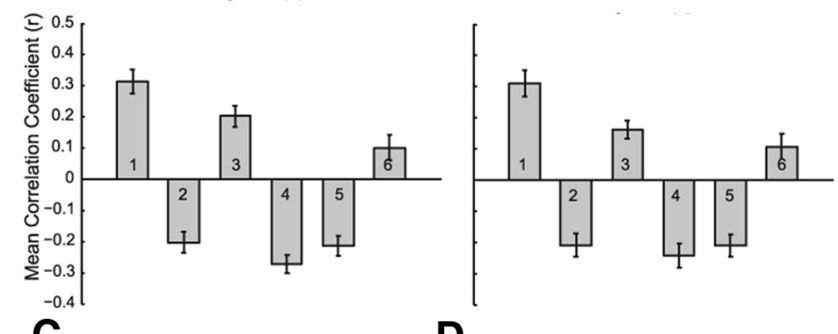

C

D
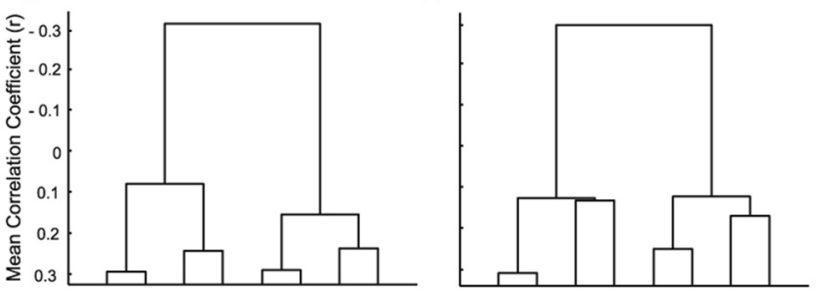

Figure 6. $\quad \boldsymbol{A}$, Representational similarity analysis $(\mathrm{RS} A)$ including only events that involved the rewarded object as the first object-sampling event in a trial. $\boldsymbol{B}, \mathrm{RSA}$ including only events that involved the rewarded object as the second object-sampling event in a trial. See description of conditions in Figure 4A. C, Dendrogram including only events that involved the rewarded object as the first object-sampling event in a trial. $\boldsymbol{D}$, Dendrogram including only events that involved the rewarded object as the second object-sampling event in a trial. Top level, Value; middle level, object-in-context; bottom level, position. See description of levels in Figure $4 B$.

The same representational pattern is apparent in each subject To assess whether ensemble representations were similar across individual animals, we made the same three comparisons for each subject as in the analysis of ensembles consisting of $>10$ neurons (Fig. 5B). All animals but one (Rat 3 ) had population representations that showed strong value coding (Fig. 5B, compare combined correlations from Bars, 1, 3, 6, and Bars 2, 4, 5; all $p<$ 0.008 ). All animals but one (Rat 2) had population representations that showed position coding (Fig. $5 B$, Bar 1 vs Bar 3, $p<$ 0.008 ). Finally, Rat 3 had population representations that showed significant object-in-context coding (Fig. 5B, Bar 3 vs Bar 6 ; $p<$ 0.008 ), and Rats 2 and 4 had marginally significant object-incontext coding in these Bonferroni-corrected tests $(p<0.05$; Fig. $5 B)$. Thus, even with very limited datasets in these analyses, and while not all of the dimensions were significantly coded in every animal, the overall pattern for each animal was remarkably similar to that of the group analysis (Fig. 4A).

\section{The same representational pattern is apparent regardless of the order of sampling events}

Since medial prefrontal neurons have been observed to be sensitive to the temporal order of events (Hyman et al., 2012), we created separate RSAs for object-sampling events only when the rewarded object was approached first and only when the same object was approached second. We compared each equivalent condition (Bars 1-6) for when the rewarded object was approached first with that of when the rewarded object was approached second, and observed no significant changes as a result of temporal order (all conditions, n.s.), indicating little effect of the order in which objects were sampled within a trial (Fig. 6). Thus, it appears that each object-sampling event is processed by OFC separately from the preceding event.

OFC neural ensembles represent a systematic organization of task dimensions

The dendrogram analysis (see Materials and Methods) is consistent with the possibility of a highly systematic structure of the 
population representation indicating successive levels of increasing representational similarity among the relevant task dimensions (Fig. $4 B$ ). At the top of the very systematic organization suggested by this analysis, and farthest apart in the ensemble representation, are events with opposite reward values. This dimension is notably reflected in an anticorrelation, indicating strong pattern separation of representations associated with different reward outcomes. At the next level is a separation of events that occur in different contexts within each reward value condition, and this dimension is observed within each reward outcome level. Notably, the associated correlation value was near zero for rewarded events, suggesting the independence of representations for rewarded events in different contexts, whereas the correlation for nonrewarded events was positive, indicating similarity in the representation of events not associated with reward (see above for a statistical comparison). Next, events that involve the same object and outcome at different positions within a context have somewhat distinct representations, and this dimension is observed below each object-context condition. This overall pattern suggests that representations of object-sampling events are organized into two actively separated networks defined by different reward values, and within each value-based network, events are categorized by the objects and the context in which they occur followed by their locations within a context. Using Catalan number generation $((2 n) ! /(n+1) ! n !)$, there could be 429 possible dendrogram outcomes, and therefore the possibility of the current systematic structure happening by chance is low.

\section{During each trial OFC ensembles evolve to represent key elements of each event \\ Context exploration}

Even before objects were sampled, OFC neurons and ensembles encoded the context that predicted object-reward assignments. To determine context selectivity for each neuron, we first performed a one-way ANOVA on firing rates during the first $5 \mathrm{~s}$ of context exploration events that preceded the presentation of the objects. This analysis revealed that $26 \%$ of recorded neurons fired differentially associated with the two contexts (Table 1). Examples shown in Figure $7 A$ show that Cell 5 fired at twice the rate in Context 1 compared with Context 2 for the entire context exploration period $\left(F_{(1,71)}=78.92, p=0.0001\right)$, and Cell 6 had the opposite preference $\left(F_{(1,69)}=29.74, p=0.0001\right)$.

In analyses of ensemble representations, RSA indicated that correlations obtained between same-context exploration events were significantly greater than those between contexts (within vs between context, $p<0.0001$; Fig. $7 B$ ).

\section{Object sampling}

To examine the evolution of position and object-reward representations after the divider was removed, allowing the rat to approach the pot, population vectors were composed for $200 \mathrm{~ms}$ time bins from $3.4 \mathrm{~s}$ before to $3.4 \mathrm{~s}$ after the onset of objectsampling events of at least $500 \mathrm{~ms}$. Ensemble coding for each time bin was measured as the difference in average correlation coefficients between the same and different events belonging to the same dimension (position or object), and the $d^{\prime}$ metric (see Materials and Methods) was used to reflect the representational difference in each comparison. Position coding was apparent well before the animal reached the object and peaked $400 \mathrm{~ms}$ before the onset of object sampling $\left(d^{\prime}=1.21, p<0.0001\right)$, then gradually declined (Fig. 8). The decrease in position coding was coincident with a sharp increase during object sampling in the coding of different objects and their associated reward values, peaking at $800 \mathrm{~ms}$ after the onset of object sampling $\left(d^{\prime}=1.82, p<0.0001\right)$.
The combined findings indicate a dynamic of population coding in which OFC ensembles first represent the context that defines object and reward associations then, before object sampling, the position of the animal, then the objects and their associated values increasingly during object sampling.

\section{Discussion}

This study examined the role of the rat orbitofrontal cortex in the retrieval of memories where the reward associations of events depend on the context in which they were experienced. The patterns of neuronal activity in OFC neurons and neuronal ensembles distinguished the contexts in which objects were associated with different reward values, then locations in which those objects were presented, and then the object-value associations. Results obtained during object-sampling events indicated that OFC networks process all of the relevant event dimensions, including the objects in each context and the locations in which they occurred, and their associated expectancy of reward. Most striking was the similarity of population representations of events associated with the same associated reward value and strong pattern separation of the OFC representations of events associated with different reward value, suggesting actively separated networks. This observation is consistent with previous reports that OFC neurons represent associations between cues and the values of reinforcement outcomes for behavioral responses to those cues (McDannald et al., 2005; Mainen and Kepecs, 2009; Schoenbaum et al., 2009, 2011; Brown et al., 2010; Walton et al., 2010; Padoa-Schioppa and Cai, 2011; Steiner and Redish, 2012). Notably, however, in the design of the present study distinct behavioral responses (dig or withdraw) were confounded with specific reward outcomes (reward or nonreward, respectively). So, we cannot unambiguously attribute the representations of reward value from the overall outcome of the trial as a particular behavioral response leading to a specific reward value.

The present findings are also consistent with reports of OFC neurons reversing their firing selectivity between stimuli when the stimulus-reward associations switch (Rolls et al., 1996; Schoenbaum et al., 1999). In context-guided memory, the stimulus-reward associations "reverse" as a function of the surrounding context in which they occur, and the dramatic change in OFC firing patterns during sampling of the same object between the two contexts reflects this context-directed reversal in reinforcement contingencies. OFC neurons not only represented the rewarded object, but also showed firing selectivity to the object that was not associated with reward within each context, consistent with previous reports of the role of this region in representing the value of the outcome associated with an event or response, regardless of its affective nature (Ostlund and Balleine, 2007; Schoenbaum et al., 2009). The present findings on firing patterns during context exploration before object sampling also extend earlier observations on OFC representation to the encoding and retrieval of abstract contextual representations, or "states," that dictate a combination of object-reward associations during the context exploration phase as well as a "mapping" of the contexts, locations, and stimuli associated with specific behavioral responses that lead to distinct rewards (Wilson et al., 2014).

Recent approaches to ensemble representation have shown that populations of neurons with complex firing patterns in dorsolateral prefrontal areas of monkeys flexibly encode multiple dimensions of experience that evolve during decision making (Mante et al., 2013; Rigotti et al., 2013; Stokes et al., 2013), and several earlier studies have identified multiple dimensions of events predicting outcomes encoded by OFC neurons in rats and monkeys (Furuyashiki and Gallagher, 2007; Wallis, 2007; Mainen and Kepecs, 2009; Schoenbaum 
A
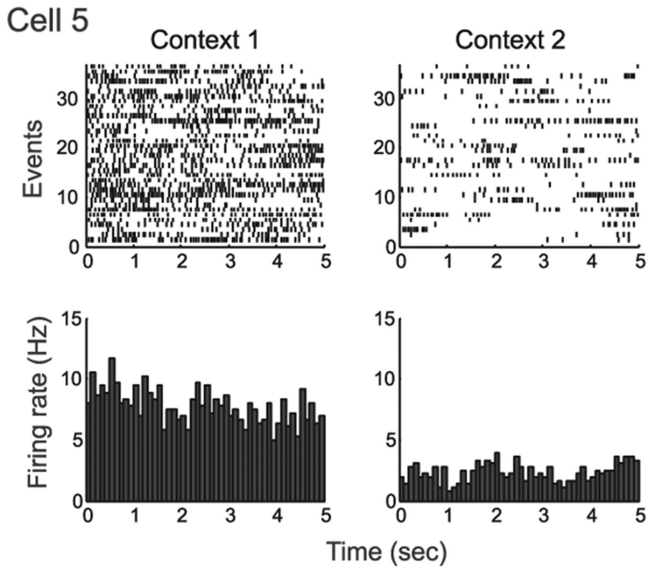

Cell 6
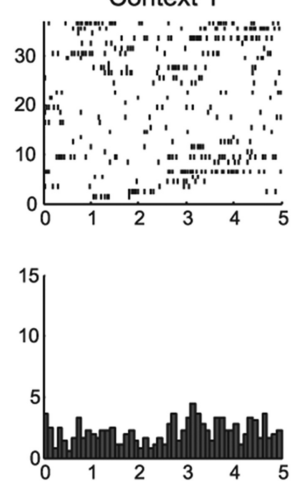

B
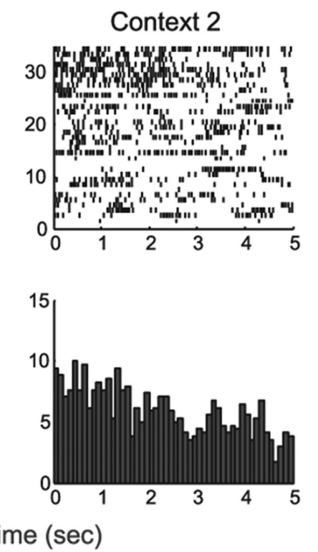

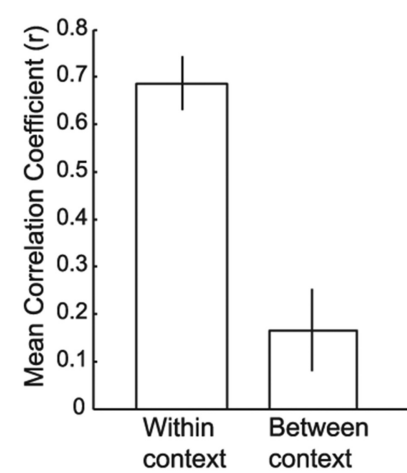

Figure 7. A, Activity of example neurons during context exploration. Cells 3 and 4 are examples of neurons whose firing patterns distinguished the two contexts. Rasters and perievent histograms depict neuronal activity during exploration within each context. Time 0 is when the nose of the animal passed the entered context. $\boldsymbol{B}$, Ensemble activity during context exploration. Within context, Cross-correlations of population vectors taken between exploration events in the same context; between context, cross-correlations of population vectors taken between explorations of different contexts. ${ }^{* * *} p<0.0001$.

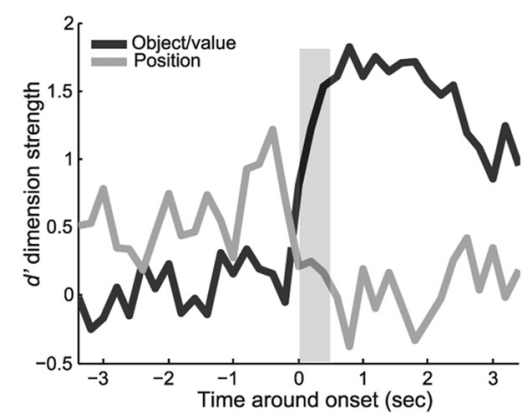

Figure 8. Dynamics of position and object-value coding around onset of object-sampling events. Correlation analysis was performed for $200 \mathrm{~ms}$ time bins starting at $-3.4 \mathrm{~s}$ before object-sampling onset and ending at $+3.4 \mathrm{~s}$ after object-sampling onset, and ensemble discrimination for each dimension was represented using the $d^{\prime}$ metric (see Materials and Methods). Light shade over the first 500 ms highlights the minimum sampling duration.

et al., 2009; Young and Shapiro, 2011). The representational similarity analysis of neural ensembles in rodent OFC applied here provides new insights into the structure of OFC network representation of multiple task dimensions in the current task, revealing a systematic organization of the relevant features of the stimuli and reward contingencies consistent with a mapping of contextual cues and specific stimuli associated with behavioral responses and the values of consequent reinforcement outcomes envisioned by Wilson et al. (2014). Events associated with opposite reward value involved strongly pattern-separated representations. This observation indicates the establishment of distinct OFC networks-or schemas - that encode events that were associated with reward and nonreward. The separation of these schemas for opposing object-reward associations could support the reduction of interference between events that share object, position, or context features but lead to opposite outcomes. Within each distinct value-based schema, objects in different contexts that predict the same outcome are independently represented, whereas distinct objects that were not associated with reward were coded as similar across contexts, suggesting that object-context representation depended on reward status. Within each context representation, events were separated by position within the context, and finally, within each position representation, events having the same object identity and value were encoded most similarly.

\section{Orbitofrontal cortex}

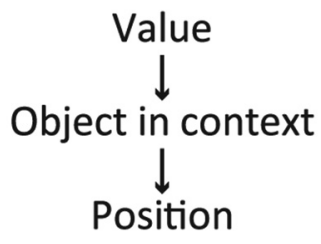

Figure 9. Systematic organization of task dimensions by orbitofrontal and dorsal hippocampal neuronal populations. Dorsal hippocampus organization based on the study of McKenzie et al. (2014).

This representational organization is strikingly different from the representation developed by the hippocampus in animals performing the same context-guided object association task (McKenzie et al., 2014; Fig. 9). The hippocampal network strongly pattern separates events by the context in which they occur, thus establishing distinct representational networks that include both rewarded and nonrewarded events that occur in different contexts. Within each distinct context-defined schema, events are separated by the locations where they occur within a context. Within representations of locations, events were separated by reward value, and within representations of reward values, events were separated by object identity. Thus, whereas the hippocampus develops a context-based schema, the OFC develops a value-based schema. The distinctive organizations of task dimensions in OFC and the hippocampus suggest commonality in the information contained in these brain areas and complementary organization of information processing relevant to this paradigm.

These observations extend our model of prefrontal-hippocampal interactions that support performance in contextguided memory (Navawongse and Eichenbaum, 2013). This model is based on the known anatomical pathways between the prefrontal cortex and hippocampus, and on findings on the firing patterns in the dorsal and ventral hippocampus. According to the model, inputs from neocortical areas that process information about object identity arrive in the hippocampus via the perirhinal 
and lateral entorhinal cortex, whereas inputs about spatial context arrive in the hippocampus via the postrhinal and medial entorhinal cortex (Eichenbaum et al., 2007). These pathways likely support a merging of object and context information within the hippocampus. Correspondingly, in animals performing the context-guided memory task studied here, within the dorsal hippocampus, neurons rapidly form representations of objects in the positions and contexts in which they occur (Komorowski et al., 2009). More gradually, neurons within the ventral hippocampus strongly distinguish events within each context but also generalize across events and locations within each context (Komorowski et al., 2013). Direct outputs from the hippocampus to both medial prefrontal cortex (mPFC) and OFC are generated in the ventral hippocampus (Jay and Witter, 1991), suggesting that ventral hippocampal context representations might be the source of information to prefrontal cortex about the events that occur in each context (although there are also alternative sources of context information to OFC, e.g., postrhinal cortex; Agster and Burwell, 2009; Kondo and Witter, 2014). These inputs may support the OFC in its development of abstract representations that map the set of events associated with different reward values within each context. During memory retrieval after learning, signals about the context occupied may be sent from ventral hippocampus to OFC, regenerating the appropriate abstract context representation observed in this study during the context exploration period. Mechanisms within OFC may then generate the appropriate elements of the systematic representation of events in OFC during object sampling, which then guides the dorsal hippocampus to retrieve appropriate object representations based on associated reward value.

Notably, it is very likely that the mPFC plays a role in this functional circuitry as well. The mPFC is essential to retrieval of context-guided memories (Peters et al., 2013), including in this task (Navawongse and Eichenbaum, 2013), and several studies have shown that the mPFC is involved in rule switching (Birrell and Brown, 2000; Floresco et al., 2008; Rich and Shapiro, 2009), as well as rule-governed behavior (Wise et al., 1996; Miller et al., 2002). In addition, rat mPFC, similar to its putative functional analog primate dorsolateral prefrontal cortex (e.g., Brown and Bowman, 2002; Wise, 2008; but see Vogt and Paxinos, 2014), has neuronal firing patterns that distinguish contexts (Hyman et al., 2012; Mante et al., 2013), and mPFC neurons respond differentially when task response demands are altered (Rich and Shapiro, 2009; Durstewitz et al., 2010). The mPFC also receives input from ventral hippocampus and is highly interconnected with OFC. Furthermore, mPFC inactivation results in loss of object-specific responses in the dorsal hippocampus (Navawongse and Eichenbaum, 2013), and the critical influence of OFC is perhaps via its direct connections with the perirhinal and lateral entorhinal cortex. The complementary influences and nature of interactions between these prefrontal areas in support of context-guided memory retrieval remain to be determined. One possibility is that mPFC and OFC may differentially support context-specific rules associated with attention or actions versus expected rewards, respectively (Sul et al., 2010).

\section{References}

Agster KL, Burwell RD (2009) Cortical efferents of the perirhinal, postrhinal, and entorhinal cortices of the rat. Hippocampus 19:1159-1186. CrossRef Medline

Balaz MA, Capra S, Hartl P, Miller RR (1981) Contextual potentiation of acquired behavior after developing direct context-US associations. Learn Mem 12:383-397. Medline

Birrell JM, Brown VJ (2000) Medial frontal cortex mediates perceptual attentional set shifting in the rat. J Neurosci 20:4320-4324. Medline

Brown TI, Ross RS, Keller JB, Hasselmo ME, Stern CE (2010) Which way was I going? Contextual retrieval supports the disambiguation of well learned overlapping navigational routes. J Neurosci 30:7414-7422. CrossRef Medline

Brown VJ, Bowman EM (2002) Rodent models of prefrontal cortical function. Trends Neurosci 25:340-343. CrossRef Medline

Delatour B, Witter MP (2002) Projections from the parahippocampal region to the prefrontal cortex in the rat: evidence of multiple pathways. Eur J Neurosci 15:1400-1407. CrossRef Medline

Durstewitz D, Vittoz NM, Floresco SB, Seamans JK (2010) Abrupt transitions between prefrontal neural ensemble states accompany behavioral transitions during rule learning. Neuron 66:438-448. CrossRef Medline

Eichenbaum H, Yonelinas AP, Ranganath C (2007) The medial temporal lobe and recognition memory. Annu Rev Neurosci 30:123-152. CrossRef Medline

Floresco SB, Block AE, Tse MT (2008) Inactivation of the medial prefrontal cortex of the rat impairs strategy set-shifting, but not reversal learning, using a novel, automated procedure. Behav Brain Res 190:85-96. CrossRef Medline

Furuyashiki T, Gallagher M (2007) Neural encoding in orbitofrontal cortex related to goal-directed behavior. Ann N Y Acad Sci 1121:193-215. CrossRef Medline

Ghods-Sharifi S, Haluk DM, Floresco SB (2008) Differential effects of inactivation of the orbitofrontal cortex on strategy set-shifting and reversal learning. Neurobiol Learn Mem 89:567-573. CrossRef Medline

Greenspoon J, Ranyard R (1957) Stimulus conditions and retroactive inhibition. J Exp Psychol 53:55-59. CrossRef Medline

Hyman JM, Ma L, Balaguer-Ballester E, Durstewitz D, Seamans JK (2012) Contextual encoding by ensembles of medial prefrontal cortex neurons. Proc Natl Acad Sci U S A 109:5086-5091. CrossRef Medline

Jay TM, Witter MP (1991) Distribution of hippocampal CA1 and subicular efferents in the prefrontal cortex of the rat studied by means of anterograde transport of Phaseolus vulgaris-leucoagglutinin. J Comp Neurol 313:574-586. CrossRef Medline

Komorowski RW, Manns JR, Eichenbaum H (2009) Robust conjunctive object-place coding by hippocampal neurons parallels learning what happens where. J Neurosci 29:9918-9929. CrossRef Medline

Komorowski RW, Garcia CG, Wilson A, Hattori S, Howard MW, Eichenbaum H (2013) Ventral hippocampal neurons are shaped by experience to represent behaviorally relevant contexts. J Neurosci 33:8079-8087. CrossRef Medline

Kondo H, Witter MP (2014) Topographic organization of orbitofrontal projections to the parahippocampal region in rats. J Comp Neurol 522: 772-793. CrossRef Medline

Kriegeskorte N, Mur M, Bandettini P (2008) Representational similarity analysis-connecting the branches of systems neuroscience. Front Syst Neurosci 2:4. CrossRef Medline

Langston RF, Wood ER (2010) Associative recognition and the hippocampus: differential effects of hippocampal lesions on object-place, objectcontext and object-place-context memory. Hippocampus 20:1139-1153. CrossRef Medline

Mainen ZF, Kepecs A (2009) Neural representation of behavioral outcomes in the orbitofrontal cortex. Curr Opin Neurobiol 19:84-91. CrossRef Medline

Mante V, Sussillo D, Shenoy KV, Newsome WT (2013) Context-dependent computation by recurrent dynamics in prefrontal cortex. Nature 503:78-84. CrossRef Medline

McAlonan K, Brown VJ (2003) Orbital prefrontal cortex mediates reversal learning and not attentional set shifting in the rat. Behav Brain Res 146: 97-103. CrossRef Medline

McDannald MA, Saddoris MP, Gallagher M, Holland PC (2005) Lesions of orbitofrontal cortex impair rats' differential outcome expectancy learning but not conditioned stimulus-potentiated feeding. J Neurosci 25:46264632. CrossRef Medline

McKenzie S, Frank AJ, Kinsky NR, Porter B, Rivière PD, Eichenbaum H (2014) Hippocampal representation of related and opposing memories develop with distinct, hierarchically organized neural schemas. Neuron 83:202-215. CrossRef Medline

Miller EK, Cohen JD (2001) An integrative theory of prefrontal cortex function. Annu Rev Neurosci 24:167-202. CrossRef Medline

Miller EK, Freedman DJ, Wallis JD (2002) The prefrontal cortex: categories, concepts and cognition. Philos Trans R Soc Lond B Biol Sci 357:11231136. CrossRef Medline

Navawongse R, Eichenbaum H (2013) Distinct pathway for rule-based retrieval and spatial mapping of memory representations in hippocampal neurons. J Neurosci 33:1002-1013. CrossRef Medline

Ostlund SB, Balleine BW (2007) The contribution of orbitofrontal cortex to action selection. Ann NY Acad Sci 1121:174-192. CrossRef Medline 
Padoa-Schioppa C, Cai X (2011) The orbitofrontal cortex and the computation of subjective value: consolidated concepts and new perspectives. Ann NY Acad Sci 1239:130-137. CrossRef Medline

Paxinos G, Watson C (2007) The rat brain in stereotaxic coordinates, Ed 6. New York: Academic, Elsevier.

Peters GJ, David CN, Marcus MD, Smith DM (2013) The medial prefrontal cortex is critical for memory retrieval and resolving interference. Learn Mem 20:201-209. CrossRef Medline

Rich EL, Shapiro M (2009) Rat prefrontal cortical neurons selectively code strategy switches. J Neurosci 29:7208-7219. CrossRef Medline

Rigotti M, Barak O, Warden MR, Wang XJ, Daw ND, Miller EK, Fusi S (2013) The importance of mixed selectivity in complex cognitive tasks. Nature 497:585-590. CrossRef Medline

Rolls ET, Critchley HD, Mason R, Wakeman EA (1996) Orbitofrontal cortex neurons: role in olfactory and visual association learning. J Neurophysiol 75:1970-1981. Medline

Rudebeck PH, Murray EA (2014) The orbitofrontal oracle: cortical mechanisms for the prediction and evaluation of specific behavioral outcomes. Neuron 84:1143-1156. CrossRef Medline

Schnider A, Ptak R (1999) Spontaneous confabulators fail to suppress currently irrelevant memory traces. Nat Neurosci 2:677-681. CrossRef Medline

Schoenbaum G, Chiba AA, Gallagher M (1999) Neural encoding in orbitofrontal cortex and basolateral amygdala during olfactory discrimination learning. J Neurosci 19:1876-1884. Medline

Schoenbaum G, Roesch MR, Stalnaker TA, Takahashi YK (2009) A new perspective on the role of the orbitofrontal cortex in adaptive behavior. Nat Rev Neurosci 10:885-892. CrossRef Medline

Schoenbaum G, TakahashiY, Liu TL, McDannaldMA (2011) Does the orbitofrontal cortex signal value? Ann N Y Acad Sci 1239:87-99. CrossRef Medline

Smith DM, Mizumori SJ (2006) Learning-related development of context- specific neuronal responses to places and events: the hippocampal role in context processing. J Neurosci 26:3154-3163. CrossRef Medline

Steiner AP, Redish AD (2012) The road not taken: neural correlates of decision making in orbitofrontal cortex. Front Neurosci 6:131. CrossRef Medline

Stokes MG, Kusunoki M, Sigala N, Nili H, Gaffan D, Duncan J (2013) Dynamic coding for cognitive control in prefrontal cortex. Neuron 78:364375. CrossRef Medline

Sul JH, Kim H, Huh N, Lee D, Jung MW (2010) Distinct roles of rodent orbitofrontal and medial prefrontal cortex in decision making. Neuron 66:449-460. CrossRef Medline

Tremblay L, Schultz W (2000) Modifications of reward expectation-related neuronal activity during learning in primate orbitofrontal cortex. J Neurophysiol 83:1877-1885. Medline

Vogt BA, Paxinos G (2014) Cytoarchitecture of mouse and rat cingulate cortex with human homologies. Brain Struct Funct 219:185-192. CrossRef Medline

Wallis JD (2007) Neuronal mechanisms in prefrontal cortex underlying adaptive choice behavior. Ann N Y Acad Sci 1121:447-460. CrossRef Medline

Walton ME, Behrens TE, Buckley MJ, Rudebeck PH, Rushworth MF (2010) Separable learning systems in the macaque brain and the role of orbitofrontal cortex in contingent learning. Neuron 65:927-939. CrossRef Medline

Wilson RC, Takahashi YK, Schoenbaum G, Niv Y (2014) Orbitofrontal cortex as a cognitive map of task space. Neuron 81:267-279. CrossRef Medline

Wise SP (2008) Forward frontal fields: phylogeny and fundamental function. Trends Neurosci 31:599-608. CrossRef Medline

Wise SP, Murray EA, Gerfen CR (1996) The frontal cortex-basal ganglia system in primates. Crit Rev Neurobiol 10:317-356. CrossRef Medline

Young JJ, Shapiro ML (2009) Double dissociation and hierarchical organization of strategy switches and reversals in the rat PFC. Behav Neurosci 123:1028-1035. CrossRef Medline

Young JJ, Shapiro ML (2011) Dynamic coding of goal-directed paths by orbital prefrontal cortex. J Neurosci 31:5989-6000. CrossRef Medline 